\title{
DIBs Broadening with Increased Abundance of Vibrationally Excited $\mathbf{H}_{2}$ Molecules
}

\author{
P. Gnaciński ${ }^{1}$ and J. Krełowski ${ }^{2}$ \\ ${ }^{1}$ University of Gdańsk, ul. Wita Stwosza 57, 80-952 Gdańsk, Poland \\ email: fizpg@univ.gda.pl \\ ${ }^{2}$ Nicolaus Copernicus University, ul. Gagarina 11, 87-100 Toruń, Poland \\ email: Jacek.Krelowski@astri.uni.torun.pl
}

\begin{abstract}
The analysis of DIB profiles was used in various attempts to discover the DIBs carriers. The broadening of the $6196 \AA$ DIB with increasing $\mathrm{C}_{2}$ rotational temperature was reported by Kaźmierczak et al. (2009). We present the broadening of the 4763, 5780 and $6614 \AA$ DIBs connected with higher abundance of vibrationally excited hydrogen molecule. The DIBs broadening is also correlated with increasing $\mathrm{H}_{2}$ rotational temperature on the $\nu=2$ vibrational level. The broadening may be caused by growing population of higher rotational levels in the DIB carrier. Some DIBs, like $4964 \AA$, do not show any width changes with higher population of vibrationally excited $\mathrm{H}_{2}$.
\end{abstract}

Keywords. ISM: molecules, ISM: lines and bands, ultraviolet: ISM

The ultraviolet radiation field from an early spectral-type star can populate the vibrationally excited $\mathrm{H}_{2}$ levels (hereafter $\mathrm{H}_{2}^{*}$ ) in interstellar clouds. The absorption spectral lines of interstellar $\mathrm{H}_{2}^{*}$ are placed in the ultraviolet spectral range observed by STIS (Space Telescope Imaging Spectrograph) onboard the HST (Hubble Space Telescope). Objects with highly populated vibrationally excited $\mathrm{H}_{2}$ levels are very rare, since such cases are restricted to interstellar clouds situated in vicinities of hot stars (the star-cloud distance must be less than $1 \mathrm{pc}$ ). An example of $\mathrm{H}_{2}^{*}$ absorption lines in the ultraviolet STIS spectrum of HD 37903 is presented in figure 1.

The observations of vibrationally excited $\mathrm{H}_{2}$ in the interstellar matter give us a rare opportunity to estimate the ultraviolet radiation flux in the interstellar clouds. We used a parameter $\mathrm{X}\left(\mathrm{H}_{2}^{*}\right)=\mathrm{N}\left(\mathrm{H}_{2}^{*}\right) / \mathrm{N}\left(\right.$ total $\left.\mathrm{H}_{2}\right)$ as an indicator of $\mathrm{UV}$ radiation field toward $\mathrm{HD}$ 37903, HD 147888 and HD 37061. The $\mathrm{H}_{2}^{*}$ toward these stars was detected by Meyer et al. (2001). The $\mathrm{H}_{2}^{*}$ column densities toward these stars were derived by Gnaciński (2009, 2011, 2013).

The spectra of all three analyzed stars show only one interstellar Doppler component in the lines of $\mathrm{K} \mathrm{I}$ and/or the $\mathrm{CH}$ molecule. The column densities of unobserved rotational levels toward HD 37061 and HD 147888 were calculated from the rotational temperatures

Table 1. Rotational temperature of para $-\mathrm{H}_{2}$ on $\nu=2$ vibrational level and relative abundance of vibrationally excited molecular hydrogen.

\begin{tabular}{lrc}
\hline star & $\begin{array}{c}\text { Para- } \mathrm{H}_{2} \text { rotational } \\
\text { temperature on } \nu=2\end{array}$ & $\begin{array}{c}\mathrm{X}\left(\mathrm{H}_{2}^{*}\right) \text { from } \\
\nu=1-5 \text { and } \mathrm{J}=0-13\end{array}$ \\
\hline HD 37061 & $2088 \pm 231 \mathrm{~K}$ & $8.0 \mathrm{E}-06 \pm 6.4 \mathrm{E}-06$ \\
HD 37903 & $1779 \pm 210 \mathrm{~K}$ & $2.4 \mathrm{E}-06 \pm 7.8 \mathrm{E}-07$ \\
HD 147888 & $791 \pm 128 \mathrm{~K}$ & $5.6 \mathrm{E}-07 \pm 9.9 \mathrm{E}-08$ \\
\hline
\end{tabular}




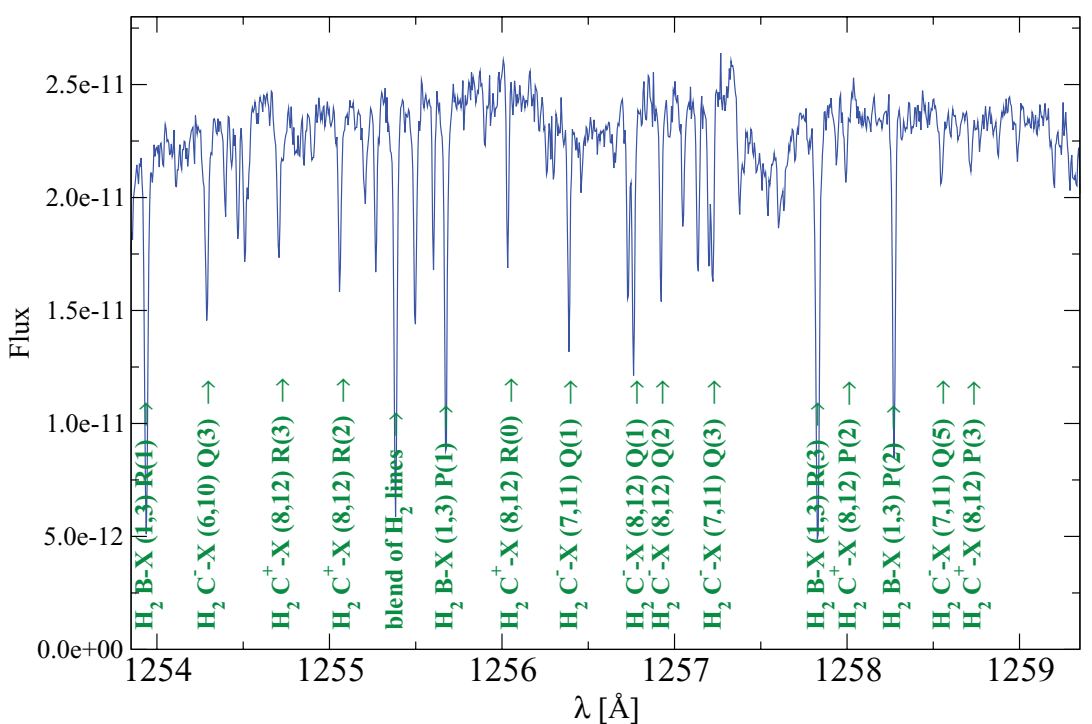

Figure 1. Fragment of HST STIS spectrum of HD 37903. All absorption lines seen on this plot are $\mathrm{H}_{2}$ lines originate from vibrationally excited levels.

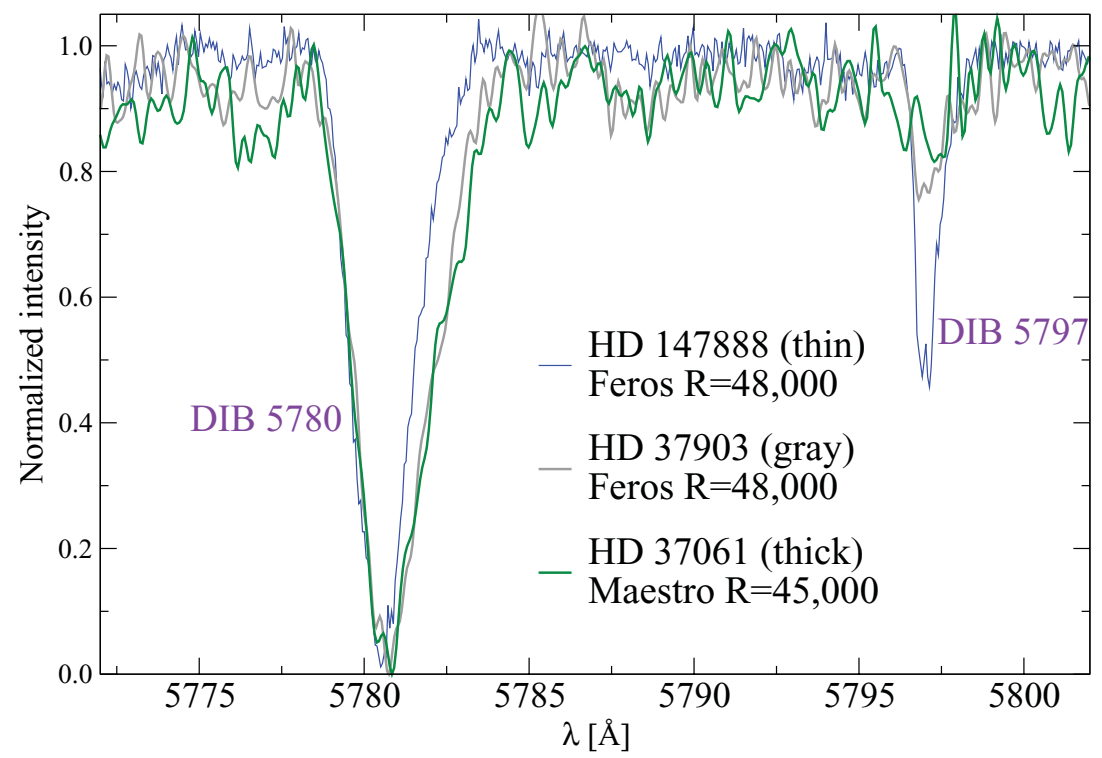

Figure 2. Broadening of the $5780 \AA$ DIB. The spectra were normalized to common depth of the $5780 \AA$ DIB.

separately for ortho- and para- $\mathrm{H}_{2}$. The column densities were calculated up to rotational number $\mathrm{J}=13$ and for vibrational levels $\nu=0-5$.

We have also calculated the rotational temperature of para- $\mathrm{H}_{2}$ on the $\nu=2$ vibrational level. The latter was chosen because it was observed toward all three objects and the errors of column densities were relatively low. The derived rotational temperature correlates with our $\mathrm{X}\left(\mathrm{H}_{2}^{*}\right)$ parameter as can be seen in Table 1. 
The objects with higher populations of vibrationally excited $\mathrm{H}_{2}^{*}$ levels (highest $\mathrm{X}\left(\mathrm{H}_{2}^{*}\right)$ ) and higher para- $\mathrm{H}_{2}$ rotational temperature on $\nu=2$ vibrational level have the DIBs 4763 , 5780 and 6614 noticeably broader (see Figure 2). Widths of other DIBs, like 4964, seem to be insensitive to the $\mathrm{X}\left(\mathrm{H}_{2}^{*}\right)$ parameter. All DIBs are relatively weak if $\mathrm{X}\left(\mathrm{H}_{2}^{*}\right)$ is high.

The star radiation that populates the vibrationally excited $\mathrm{H}_{2}^{*}$ levels seems also to populate higher rotational levels in the DIBs carriers. The sensitivity of DIBs to the $\mathrm{X}\left(\mathrm{H}_{2}^{*}\right)$ parameter may lead to distinguish DIB families with common reaction to the radiation field.

\section{Acknowledgements}

Based on observations made with ESO Telescopes at the La Silla Paranal Observatory under programme ID 076.C-0164(A) and 076.C-0431(A) and on observations made using the Maestro spectrograph of the Terskol Observatory (Russia) fed with the $2 \mathrm{~m}$ telescope. The authors are very grateful to the staff members of these observatories for the experienced help. This work was supported by NCN (Polish National Science Center) grants DEC-2012/05/D/ST9/03912 and 5820/B/H03/2011/40.

\section{References}

Gnaciński, P. 2009, Acta Astron., 59, 325

Gnaciński, P. 2011, A\&A, 532, A122

Gnaciński, P. 2013, A\&SA, 549, A37

Kaźmierczak, M., et al. 2009, A\&\&A, 498, 785

Meyer, D., et al. 2001, ApJ, 553, L59 